\title{
Wolbachia in the spittlebug Prosapia ignipectus: Variable infection frequencies, but no apparent effect on host reproductive isolation
}

\author{
Timothy B. Wheeler ${ }^{\dagger}$, Vinton Thompson ${ }^{\S}$, William R. Conner ${ }^{\dagger}$, and Brandon S. Cooper ${ }^{\dagger}{ }^{*}$ \\ ${ }^{\dagger}$ Division of Biological Sciences \\ University of Montana \\ 32 Campus Dr. \\ Missoula, MT 59812 \\ ${ }^{\S}$ Division of Invertebrate Zoology \\ American Museum of Natural History \\ Central Park West at 79 ${ }^{\text {in }}$ St. \\ New York, NY 10024 \\ *Corresponding Author \\ Brandon S. Cooper \\ Division of Biological Sciences, University of Montana \\ 32 Campus Dr., Missoula, MT 59812 \\ brandon.cooper@umontana.edu
}

Keywords: Cercopidae, cytoplasmic incompatibility, endosymbiosis, host-microbe interaction, speciation 


\section{Abstract}

2 Animals serve as hosts for complex communities of microorganisms, including

3 endosymbionts that live inside their cells. Wolbachia bacteria are perhaps the most common

4 endosymbionts, manipulating host reproduction to propagate. Many Wolbachia cause intense

5 cytoplasmic incompatibility (CI) that promotes their spread to high and relatively stable

6 frequencies. Wolbachia that cause weak or no CI tend to persist at intermediate, often variable,

7 frequencies. Wolbachia could also contribute to host reproductive isolation (RI), although

8 current support for such contributions is limited to a few systems. To test for Wolbachia

9 frequency variation and effects on host RI, we sampled several local Prosapia ignipectus

10 (Fitch)(Hemiptera: Cercopidae) spittlebug populations in the northeastern USA over two years,

11 including closely juxtaposed Maine populations with different monomorphic color forms,

12 "black" and "lined". We discovered a group-B Wolbachia (wPig) infecting P. ignipectus that

13 diverged from group-A Wolbachia-like model wMel and wRi strains in Drosophila-6 to 46

14 MYA. Populations of the sister species Prosapia bicincta (Say) from Hawaii and Florida are

15 uninfected, suggesting that P.ignipectus acquired $w$ Pig after their initial divergence. $w$ Pig

16 frequencies were generally high and variable among sites and between years. While phenotyping

$17 w$ Pig effects on host reproduction is not currently feasible, the $w$ Pig genome contains three

18 divergent sets of CI loci, consistent with high $w$ Pig frequencies. Finally, Maine monomorphic

19 black and monomorphic lined populations of $P$. ignipectus share both $w$ Pig and mtDNA

20 haplotypes, implying no apparent effect of $w$ Pig on the maintenance of this morphological

21 contact zone. We hypothesize $P$. ignipectus acquired $w$ Pig horizontally as observed for many

22 Drosophila species, and that significant CI and variable transmission produce high but variable

$23 w$ Pig frequencies. 


\section{Introduction}

Animals interact with microorganisms that influence their behavior, physiology, and

34 fitness (Hurst and Jiggins, 2000; Brownlie et al., 2009; McFall-Ngai et al., 2013; Fredericksen et al., 2017; Gould et al., 2018; Hague, Caldwell and Cooper, 2020). These include associations

36 between hosts and vertically transmitted endosymbionts that live inside their cells (McCutcheon,

37 Boyd and Dale, 2019). Hosts may acquire endosymbionts cladogenically from common ancestors (Raychoudhury et al., 2009; Koga et al., 2013; Toju et al., 2013), from sister species via hybridization and introgression (Turelli et al., 2018; Cooper et al., 2019), or horizontally in ways that are not fully understood (O’Neill et al., 1992; Huigens et al., 2000; Ahmed et al., 2015). While few examples exist, endosymbionts can contribute to host reproductive isolation (RI) and speciation (Coyne and Orr, 2004; Matute and Cooper, 2021), highlighting the importance of discovering and characterizing endosymbiont-host associations.

Maternally transmitted Wolbachia bacteria are widely distributed (Werren, Baldo and Clark, 2008; Zug and Hammerstein, 2012; Weinert et al., 2015), infecting many arthropods and two groups of parasitic nematodes (Bandi et al., 1998), making Wolbachia perhaps the most common endosymbiont in nature. In Drosophila, introgressive and horizontal Wolbachia acquisition seem to predominate (Conner et al., 2017; Turelli et al., 2018; Cooper et al., 2019), but cladogenic acquisition during host speciation has been observed in other taxa (Raychoudhury et al., 2009; Gerth and Bleidorn, 2017). Many Wolbachia manipulate host reproduction to propagate in host populations. For example, many strains cause cytoplasmic incompatibility (CI) that reduces the egg hatch of uninfected embryos fertilized by Wolbachia-infected sperm (Hoffmann and Turelli, 1997). However, if females are also infected, the embryos survive, "rescuing" CI and promoting Wolbachia spread to high frequencies (Hoffmann, Turelli and Harshman, 1990; Turelli and Hoffmann, 1995; Barton and Turelli, 2011; Kriesner et al., 2013).

Wolbachia may contribute to host RI (Coyne and Orr, 2004; Matute and Cooper, 2021), with the best evidence coming from Drosophila. Wolbachia contribute to assortative mating and postzygotic isolation between co-occurring D. paulistorum semi-species (Miller, Ehrman and Schneider, 2010), and to reinforcement of isolation between uninfected D. subquinaria and

60 Wolbachia-infected D. recens (Shoemaker, Katju and Jaenike, 1999; Jaenike et al., 2006). In

61 contrast, Wolbachia do not contribute to RI in the D. yakuba clade, which includes $w$ Yak62 infected D. yakuba, wSan-infected D. santomea, and wTei-infected D. teissieri (Cooper et al., 
2017). Thus, while some results from Drosophila strongly support contributions of Wolbachia to

64 RI, and interest in the possibility of such effects remains high, it is unknown whether Wolbachia

65 effects on RI are common in nature.

Wolbachia frequencies differ significantly among infected host taxa, ranging from very

67 low to obligately fixed infections (Bandi et al., 1998; Kriesner et al., 2013; Cooper et al., 2017;

68 Miller, Ehrman and Schneider, 2010). Wolbachia effects on reproduction (e.g., CI) and fitness, in

69 combination with imperfect maternal transmission, govern its frequencies in host populations

70 (Caspari and Watson, 1959; Hoffmann, Turelli and Harshman, 1990). Intensive sampling of a

71 few systems has revealed both stable and variable Wolbachia frequencies within host

72 populations. Wolbachia that cause intense CI, like $w$ Ri in Drosophila simulans, persist at high

73 and relatively stable frequencies, balanced by imperfect maternal transmission (Kriesner $e t$ al.,

74 2013; Turelli et al., 2018). In contrast, Wolbachia that cause weak or no CI tend to occur at

75 variable intermediate frequencies (Hoffmann, Clancy and Duncan, 1996; Hamm et al., 2014;

76 Kriesner et al., 2016; Cooper et al., 2017; Meany et al., 2019). These include wMel-like

77 Wolbachia frequencies that vary spatially in D. melanogaster and D. yakuba (Kriesner et al.,

78 2016; Hague et al., 2020), and temporally in D. yakuba and D. santomea (Cooper et al., 2017;

79 Hague, Caldwell and Cooper, 2020). In all but a few systems, limited sampling has left a gap in

80 knowledge about whether Wolbachia frequency variation is common (Hughes et al., 2011;

81 Hamm et al., 2014; Cattel et al., 2016; Schuler et al., 2016; Ross et al., 2020).

82 Prosapia ignipectus (Fitch) (Hemiptera: Cercopidae) is one of about 14 species of

83 Prosapia and one of two commonly found in the USA, the other being its sister species $P$.

84 bicincta (Say)(Hamilton, 1977). P. ignipectus occurs in southern Ontario, Canada and the

85 northeastern USA from Minnesota to Maine (Hamilton, 1977, 1982; Peck, 1999; Carvalho and

86 Webb, 2005; Thompson and Carvalho, 2016). These species vary in male genital morphology

87 and in associations with host plants, with P. ignipectus monophagous on the late season C4

88 perennial grass Schizachyrium scoparium (Little bluestem) (Hamilton, 1982; Thompson, 2004)

89 and $P$. bicincta polyphagous on a variety of C4 grasses, but not including Little bluestem (Fagan

90 and Kuitert, 1969; Thompson, 2004). Both species have conspicuous dorsal coloration, standing

91 out against their respective host plants. All $P$. bicincta individuals have a single narrow

92 transverse orange line across the widest part of the pronotum and a pair of narrow orange lines

93 across the elytra. Most $P$. ignipectus individuals have a solid black dorsal surface, but in Maine 
94 some P. ignipectus have P. bicincta-like coloration (Figure 1). Notably, only $10 \mathrm{~km}$ separate

95 monomorphic black and monomorphic lined P.ignipectus populations in western Maine, with

96 little evidence of a hybrid zone and no obvious physical barriers to mixing across the boundary

97 (Thompson and Carvalho, 2016). This morphological contact zone has persisted for at least 90

98 years. About $45 \mathrm{~km}$ southwest of this abrupt transition between aposematic color forms, three

99 other P. ignipectus populations were found to be polymorphic with both black and lined forms-

100 these populations are surrounded by monomorphic black populations. It has been hypothesized

101 that Wolbachia determined RI may contribute to preservation of the sharp Maine morphological

102 contact zone (Thompson and Carvalho, 2016).

103 Here, we use collections of P. ignipectus from several sites in the northeastern USA

104 across two years, in combination with collections of $P$. bicincta from Hawaii and Florida, USA,

105 to assess modes of Wolbachia acquisition and to test for Wolbachia frequency variation through

106 space and time. By sampling monomorphic black and lined populations and typing both

107 Wolbachia and mtDNA haplotypes, we also test for contributions of Wolbachia to host RI.

108 Finally, we generate whole genome Wolbachia data for phylogenetic analysis and to search for

109 loci associated with inducing and rescuing CI (Beckmann, Ronau and Hochstrasser, 2017;

110 LePage et al., 2017; Shropshire et al., 2018).
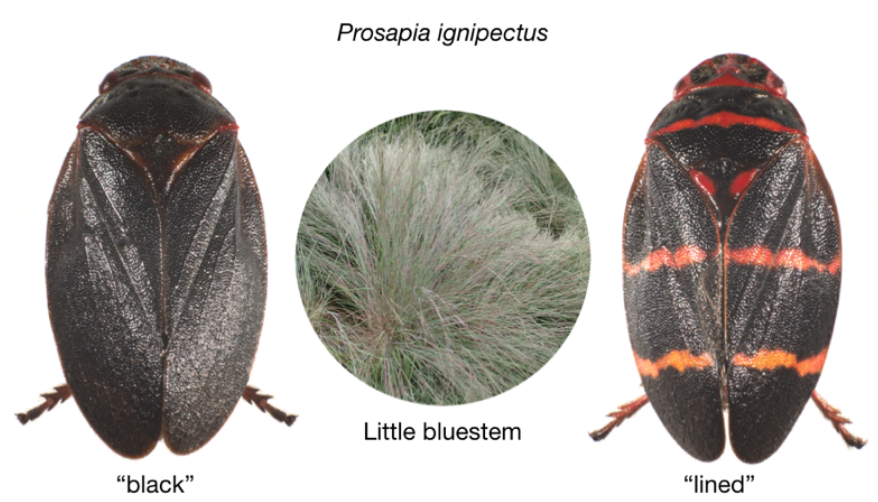

111

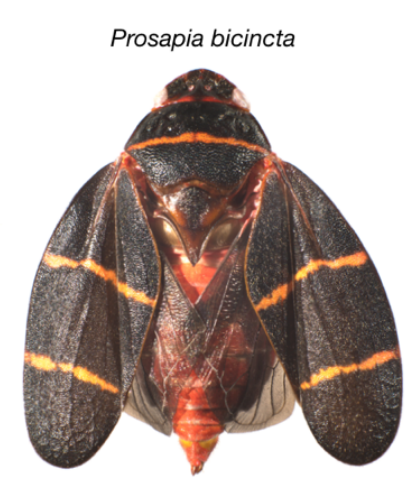

Figure 1. Sister species $P$. ignipectus and $P$. bicincta have conspicuous dorsal coloration. All P. bicincta individuals have a single narrow transverse orange line across the widest part of the pronotum and a pair of narrow orange lines across the elytra. Most $P$. ignipectus individuals have a solid black dorsal surface, but in Maine some $P$. ignipectus have $P$. bicincta-like coloration. $P$. ignipectus monophagous on the late season $\mathrm{C} 4$ perennial grass Schizachyrium scoparium (Little bluestem). Little bluestem photo by Krzysztof Ziarnek, Kenraiz (CC BY-SA 4.0, https://creativecommons.org/license s/by-sa/4.0). 


\section{Methods}

\section{Sampling}

114 We netted specimens from Little bluestem; sorted them by species, sex, and color form;

115 and preserved them in 95\% ethanol. The 2019 specimens $(N=4$ sites) were collected on August

116 23. The 2020 specimens ( $N=9$ sites) were collected on August 9 (Silver Lake, NH), August 17

117 (Wonalancet, NH), and August 20 (all Maine localities) (Supplemental Table 1). Collection sites

118 were on the verges of public rights of way or privately owned land. In two cases (New Vineyard

119 and New Portland) they correspond to sites reported in Thompson and Carvalho (2016).

120 Specimens were collected near the height of abundance for P.ignipectus, which starts to emerge

121 in adult form in late July and early August. We also sampled three additional spittlebug species

122 at these sites: Lepyronia quadrangularis (Say) $(N=25)$, Philaenus spumarius $(\mathrm{L}).(N=5)$, and

123 Philaenarcys killa (Hamilton) $(N=24)$, all of the family Aphrophoridae. Like, P. ignipectus, $P$.

124 killa a is monophage on Little bluestem. L. quadrangularis is a polyphage but often abundant on

125 Little bluestem. P. spumarius is an extreme polyphage, with a preference for forbes (herbaceous

126 perennial dicots) but is occasionally collected from Little bluestem in the company of $P$.

127 ignipectus. By screening them for Wolbachia we tested for the possibility of horizontal

128 Wolbachia transfer through plant interactions (Chrostek et al., 2017). Lastly, because

129 identification of infections in sister hosts enables formal analysis of modes of Wolbachia

130 acquisition (Turelli et al., 2018; Conner et al., 2017; Cooper et al., 2019; Raychoudhury et al.,

131 2009), we also obtained samples of the sister species $P$. bicincta from Hawaii $(N=60)$ and

132 Florida $(N=40)$ to screen for infections. $P$. bicincta is native to the southeastern USA (Fagan

133 and Kuitert, 1969; Thompson and Carvalho, 2016), but has recently been introduced into the

134 Kona Region of Big Island, Hawaii (Thorne et al., 2018).

\section{Wolbachia typing}

137 We generated whole genome Wolbachia data to type the Wolbachia infecting $P$.

138 ignipectus and to search for loci associated with CI. We extracted 800ng of high molecular

139 weight DNA (Qiagen Genomic-tip 20/G; Qiagen, Germany) from one black New Vineyard

140 female (see below), and then input and sequenced it (Ligation Sequencing Kit, SQK-LSK109;

141 FLO-MIN106 flow cell) for 48 hours (Oxford Nanopore Technologies). We mapped raw

142 nanopore reads (5.8Gb of data) to all known Wolbachia sequences (NCBI taxid 953) with 
143 BLASTn and extracted reads where at least $60 \%$ of their length mapped (qcovs $>=60$ ). We then

144 corrected and assembled reads using canu 2.1.1 (Koren et al., 2017, 2018; Nurk et al., 2020) and

145 polished the Wolbachia assembly using nanopolish 0.13.2 (Loman, Quick and Simpson, 2015).

146 We annotated our Wolbachia assembly plus the genomes of model group-A (wMel, Wu et al.,

147 2004; and wRi, Klasson et al., 2009) and group-B (wPip-Pel, Klasson et al., 2008; and wMau,

148 Meany et al., 2019) strains using Prokka v.1.11 (Seemann, 2014). We used only genes present in

149 single copy and with identical lengths in all genomes. To assess the quality of our assembly, we

150 excluded $w$ Pig and repeated this with only $w \mathrm{Mel}, w \mathrm{Ri}, w \mathrm{Pip}$, and $w \mathrm{Mau}$.

151 Preliminary analysis of a few loci placed the P. ignipectus Wolbachia in group-B (see

152 below), but we performed Bayesian analyses using the GTR + + + I model for sequence

153 evolution using whole genome data to confirm this (Höhna et al., 2016). Genes were

154 concatenated and partitioned by codon position, with a rate multiplier, $\sigma$, assigned to each

155 partition to accommodate variable substitution rates. We used flat, symmetrical Dirichlet priors

156 on the stationary base frequencies, $\pi$, and the relative-rate parameters, $\eta$, of the GTR model (i.e.,

157 Dirichlet $(1,1,1 \ldots))$. As in Turelli et al. (2018), we used a $\Gamma(2,1)$ hyperprior on the shape

158 parameter, $\alpha$, of the discrete- $\Gamma$ model (adopting the conventional assumption that the $\beta$ rate

159 parameter equals $\alpha$, so that the mean rate is 1 ; (Yang, 1994). The $\Gamma$ model for rate variation

160 assigns significant probability near zero when the $\alpha<1$ (accommodating invariant sites). The

$161 \Gamma(2,1)$ hyperprior on $\alpha$ assigns $95 \%$ probability to the interval $(0.36,4.74)$, allowing for small

162 and large values. Four independent runs for each gene set produced concordant topologies. We

163 diagnosed MCMC performance using Tracer 1.7 (Rambaut et al., 2014).

\section{Wolbachia and mtDNA haplotyping of black and lined color morphs}

166 To confirm that the same Wolbachia strain infects different P. ignipectus populations and

167 color morphs, we amplified and Sanger sequenced five protein-coding Wolbachia genes (coxA,

$168 h c p A, f b p A, f t s Z$, and $w s p$ ) in both directions (Eurofins Genomics LLC, Louisville,

169 Kentucky)(see below, Supplemental Table 2). We also amplified and Sanger sequenced gatb, but

170 sequence quality was consistently too low to include in our analyses. Samples included one

171 infected female of each color form (black or lined), from each of the four populations (Carthage,

172 New Portland, New Vineyard, and Strong) sampled in both years (Supplemental Table 1). 
To specifically assess if Wolbachia might contribute to the morphological contact zone

174 between New Vineyard (monomorphic black) and New Portland (monomorphic lined) $P$.

176 mitochondrial locus from one male and one female from these populations, with the exception of

177 one (New Vineyard black male) that did not produce usable sequence. We also produced CoI

178 sequences for one black and one lined female from the polymorphic Strong population.

We visually inspected each sequence for quality and ambiguities, and consensus

180 sequences were used as queries for a BLASTn search and the NCBI "nr" database to confirm

181 that orthologous genes were amplified (Altschul et al., 1990). We then used the "multiple locus

182 query" function of the multi locus sequence typing (MLST) database to type Wolbachia (Baldo

183 et al., 2006). Together these data enable us to test for differentiation in Wolbachia and mtDNA

184 between populations and color forms, including between populations monomorphic for different

185 color forms separated by only $10 \mathrm{~km}$ in Maine.

\section{Analysis of CI loci}

Recent work has identified CI-causing factors (cifs) associated with WO prophage in Wolbachia genomes (Beckmann, Ronau and Hochstrasser, 2017; LePage et al., 2017; Shropshire et al., 2018; Shropshire and Bordenstein, 2019; Shropshire, Leigh and Bordenstein, 2020). Two genes $($ cifA/B) transgenically expressed in male $D$. melanogaster induce CI, while one gene (cifA) expressed in females rescues it. To identify cif loci, we used BLASTn to search for cif

194 in $w \mathrm{Ri}$, the Type 3 pair in $w$ No, the Type 4 pair in $w$ Pip, and the Type 5 pair in $w$ Stri (Lindsey et 195 al., 2018; Bing et al., 2020; Martinez et al., 2020). We later broadened our search for Type 1 196 pairs by querying $w$ Pip and $w$ NPa pairs (Klasson et al., 2008; Gerth and Bleidorn, 2017). For

197 each Type, we extracted raw reads that covered at least $40 \%$ of the genes. We then corrected and 198 assembled the reads with canu 2.1.1 (Koren et al., 2017, 2018; Nurk et al., 2020), producing 199 sequences with about a 1\% error rate. We limit our analyses to the discovery of cif types, since 200 we did not generate additional sequence data to further correct the long reads. The assembled 201 genes were compared to those in Martinez et al. (2020). 


\section{Analysis of Wolbachia frequency variation}

To test for Wolbachia frequency variation, we extracted DNA from many individuals from each collection using a standard squish buffer protocol and identified Wolbachia infections using polymerase chain reaction (PCR) (Simpliamp ThermoCycler; Applied Biosystems, Singapore) (Meany et al., 2019). We amplified the Wolbachia surface protein (wsp) (Braig et al., 1998) and arthropod-specific 28S rDNA, which served as a positive control (Baldo et al., 2006) binomial distribution, we estimated exact $95 \%$ confidence intervals for Wolbachia frequencies

212 for each collection. We used Fisher's exact test (FET) to determine differences in frequencies 213 among sites, between years, between sexes, and between color forms.

Results

\section{$P$. ignipectus likely acquired its group-B Wolbachia following initial divergence from $P$.} bicincta

Across all samples, Wolbachia infection frequency $(p)$ in P. ignipectus is high $(p=0.93$

221 MLST database supports that a group-B strain, most closely related to Wolbachia in Chloropidae

222 (Diptera) (ID 93, ST 104), infects our P. ignipectus samples - we call this strain $w$ Pig.

223 Preliminary phylogenetic analyses using only our five Sanger sequenced genes also placed $w$ Pig

224 in group B. Our draft $w$ Pig assembly size $(1.32 \mathrm{Mb}, \mathrm{N} 50=91,011)$ falls in the range of complete

225 Wolbachia genomes (e.g., $w \mathrm{Mel}$ at $1.26 \mathrm{Mb}$ and $w \mathrm{Ri}$ at $1.44 \mathrm{Mb})$, despite its fragmentation $(50$

226 contigs). In total, we extracted 65 single-copy homologs of equal length (43,473 total bp) for our

227 phylogenetic analysis, which also places $w$ Pig in group B (Figure 2). When excluding the $w$ Pig

228 genome, we were able to extract an additional 135 homologs (16,7241 bp) from $w \mathrm{Mel}, w \mathrm{Ri}$,

$229 w$ Pip, and $w$ Mau. This indicates that significant residual error in the $w$ Pig assembly reduces the

230 number of homologs meeting our equal length criteria for inclusion. Finer placement of $w$ Pig

231 among group-B strains will require the generation of short-read data to further correct our draft

$232 w$ Pig assembly. Thus, we do not attempt to place $w$ Pig precisely among group-B strains. 


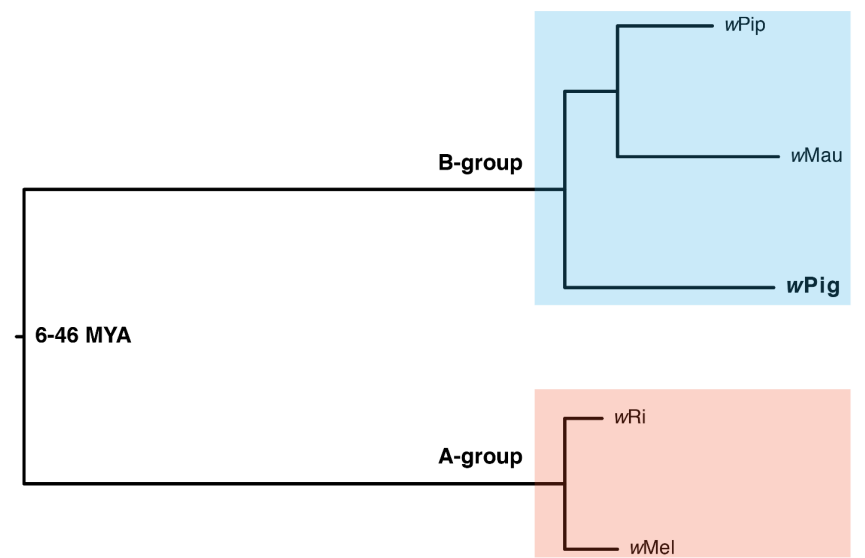

Figure 2. An estimated phylogram for model group-A (wRi, Klasson et al., 2009); and (wMel, Wu et al., 2004) and group-B (wPip_Pel, Klasson et al., 2008); and (wMau, Meany et al., 2019) Wolbachia, plus wPig. All nodes have Bayesian posterior probabilities of 1 . The divergence time of groups A and B is superimposed from (Meany et al., 2019). The phylogram shows significant variation in the substitution rates across branches, with long branches separating groups $\mathrm{A}$ and $\mathrm{B}$.

None of the $P$. bicincta samples from Hawaii and Florida were Wolbachia infected. Even if some P. bicincta are Wolbachia infected, as previously reported for one individual used as a PCR control in another study (Anderson, Rustin and Eremeeva, 2019), Wolbachia infection frequency $(p)$ must be very low across the $P$. bicincta range, given our species estimate and credible interval $(p=0.0[0.0,0.04] ; N=100)$, keeping in mind the possibility that the Hawaiian population may have experienced a recent bottleneck during introduction and may not be representative of the species in the native range. Very low frequency Wolbachia infections in global $P$. bicincta populations, in combination with generally high $w$ Pig frequencies in $P$. ignipectus, indicates that $P$. ignipectus likely acquired $w$ Pig after its initial divergence from $P$. bicincta. Because testing predictions about modes of Wolbachia acquisition requires formal analysis of Wolbachia, host nuclear, and host mtDNA phylograms and chronograms, we are unable to distinguish between introgressive and horizontal $w$ Pig transfer (Raychoudhury et al., 2009; Conner et al., 2017; Gerth and Bleidorn, 2017; Turelli et al., 2018; Cooper et al., 2019). We discuss this further below. uninfected $(p=0.0[0.0,0.14] ; N=25)$, all $P$. spumarius were infected $(p=1.0[0.48,1.0] ; N=$ 5), and only one $P$. killa individual was infected ( $p=0.04[0.001,0.21] ; N=24)$. Because

\section{3 typed the Wolbachia infecting $P$. spumarius to determine if a $w$ Pig-like variant also infects this}

254 host species. The multiple sequence query in the MLST database supports that a different group255 B strain, most closely related to the thrip species Aptinothrips rufus (ID 1945, ST 509) infects $P$. spumarius. Generating more sequence data will be required to resolve the phylogenetically 
relationships of these and other group-B strains, including Wolbachia in P. spumarius (Lis,

258 Maryańska-Nadachowska and Kajtoch, 2015).

No apparent effect of $w$ Pig on the maintenance of the morphological $P$. ignipectus contact

zone

The Strong, Carthage, and Dixfield P. ignipectus populations (Figure 3) were

262 polymorphic for the black and lined forms (Figure 1, Supplemental Table 1), like three

263 populations close to Rumford, Maine sampled in earlier work (Thompson and Carvalho, 2016).

264 This set of mixed color-form populations runs roughly from Rumford northeast to Strong, but

265 not to the sharp boundary dividing the monomorphic black New Vineyard population form the

266 monomorphic lined New Portland population. It has the appearance of a hybrid zone, but one

267 that does not reach the definitive boundary between the forms. The existence of distinct color

268 forms both within and between the populations sampled facilitated investigation of the

269 relationship, if any, between Wolbachia infection and patterns of color form occurrence.

Table 1. $w$ Pig infection frequencies in P. ignipectus at each sampled site across both years.

\begin{tabular}{lcrrr}
\hline Site & $\begin{array}{c}\text { GPS } \\
\text { coordinates }\end{array}$ & $\boldsymbol{N}$ & Infected & \multicolumn{1}{c}{$\begin{array}{c}\boldsymbol{p} \text { [Confidence } \\
\text { Interval] }\end{array}$} \\
\hline Carthage & $443644 \mathrm{~N}, 702810 \mathrm{~W}$ & 116 & 98 & $0.84[0.77,0.91]$ \\
New Portland & $445217 \mathrm{~N}, 700700 \mathrm{~W}$ & 72 & 68 & $0.94[0.86,0.98]$ \\
New Vineyard & $444514 \mathrm{~N}, 700801 \mathrm{~W}$ & 77 & 67 & $0.87[0.77,0.94]$ \\
Strong & $444708 \mathrm{~N}, 701342 \mathrm{~W}$ & 69 & 68 & $0.99[0.92,1.0]$ \\
Silver Lake & $435301 \mathrm{~N}, 711041 \mathrm{~W}$ & 20 & 19 & $0.95[0.75,1.0]$ \\
Dixfield & $443410 \mathrm{~N}, 702721 \mathrm{~W}$ & 41 & 41 & $1.0[0.91,1.0]$ \\
Weld & $444127 \mathrm{~N}, 702530 \mathrm{~W}$ & 33 & 32 & $0.97[0.84,1.0]$ \\
Wilton & $443758 \mathrm{~N}, 701810 \mathrm{~W}$ & 26 & 26 & $1.0[0.87,1.0]$ \\
Wonalancet & $435438 \mathrm{~N}, 712129 \mathrm{~W}$ & 32 & 31 & $0.97[0.84,1.0]$ \\
\hline
\end{tabular}

Sample sizes $(N)$, infection frequencies $(p)$, and exact $95 \%$ binomial confidence intervals for each site.

We found no evidence for $w$ Pig genetic differentiation between P. ignipectus populations

273 or color forms. Regions of the five $w$ Pig genes we sequenced were identical, except for a single

274 nucleotide position in wsp, where the Strong lined sample differed from all others. In addition to

275 populations sharing $w$ Pig type based on MLST loci, $w$ Pig frequency did not vary between color 
forms (black: $p=0.93[0.90,0.95], N=338$; lined: $p=0.92[0.86,0.96], N=123$; FET, $P=$ 0.69 ), among only males (black: $p=0.84[0.75,0.90], N=98$; lined: $p=0.90[0.79,0.97], N=$ 51; FET, $P=0.33$ ), or among females (black: $p=0.97$ [0.94, 0.99], $N=240$; lined: $p=0.93$ $[0.85,0.98], N=72$; FET,$P=0.19)$, across all samples. $w$ Pig frequency also did not differ

280 between New Vineyard (monomorphic black) and New Portland (monomorphic lined)

281 populations (FET, $P=0.16)$.

We found no evidence for differentiation in CoI mtDNA haplotype between the New Vineyard and New Portland P. ignipectus populations, where all samples were identical across the $680 \mathrm{bp}$ that we recovered. The black and lined females from the polymorphic Strong population also did not differ from each other, or from other populations, across this region. Thus, $w$ Pig and mtDNA haplotypes were not differentiated between populations or color forms.. Barcode of Life Database (BOLD) (Foottit, Maw and Hebert, 2014). A single base-pair insertion present in all of our samples is absent from all ten BOLD samples. Four other sites in CoI that are polymorphic among the BOLD samples are fixed in our samples for one of the BOLD alleles. mtDNA haplotypes of $P$. ignipectus and $P$. bicincta also differ by less than 2 percent

292 (Foottit, Maw and Hebert, 2014).

\section{The $w$ Pig genome contains three divergent types of CI loci}

We identified Type 1, 3, and 4 cifs in the wPig genome (Martinez et al., 2020). This specific complement of cifs is not found in any other published Wolbachia genomes, but close relatives to each $w$ Pig cif Type are. For instance, the $w$ Pig Type 1 genes are $99 \%$ identical to those in the genome of the Wolbachia infecting the gall-inducing wasp Diplolepis spinosa (Cynipidae), but less than $90 \%$ similar to any others (Martinez et al., 2020). The Type $3 w$ Pig genes are $99 \%$ identical to those in the genome of the Wolbachia infecting D. spinosa, the Staphylinid beetle Diploeciton nevermanni, and the water strider Gerris buenoi. The wPig Type 4 genes are $99 \%$ identical to those in Wolbachia infecting Nomada bees ( $w$ NLeu, wNFla, and $w \mathrm{NPa}$ ), but less than $95 \%$ identical to other Type 4 cifs. The Wolbachia infecting D. spinosa does not have Type 4 cifs, distinguishing it from $w$ Pig. None of the $w$ Pig cifs are truncated relative to copies with $99 \%$ identity. Additional sequencing is required to make more detailed cif comparisons. 


\section{Pervasive $w$ Pig frequency variation}

$w$ Pig varied in frequency in several ways. First, frequency varied spatially among all samples (FET,$P=0.001)$ (Table 1$)$, among sites in 2019 (FET,$P<0.0001)$, and 2020 (FET,$P=$ 0.033). This variation occurred over a geographic radius of only $20 \mathrm{~km}$ in 2019 and $70 \mathrm{~km}$ in 2020 (Figure 3). Second, frequency varied across all samples between $2019(p=0.88[0.82$, $0.92] ; N=169)$ and $2020(p=0.95$ [0.92, 0.97]; $N=317)($ FET,$P=0.003)$. For the four sites we sampled in both years, frequencies were only significantly different between 2019 ( $p=0.73$ $[0.56,0.86] ; N=37)$ and $2020(p=1.0[0.91,1.0] ; N=40)$ in New Vineyard (FET, $P<0.001)$.

Third, across all samples $w$ Pig frequency was higher in females $(p=0.95[0.93,0.97] ; N=332)$ than males $(p=0.86[0.80,0.91] ; N=154)($ FET,$P=0.001)$. However, this was driven mostly by a paucity of infected males in New Vineyard (males: $p=0.69$ [0.50,0.84], $N=32$; females: $p$

$318=1.0[0.92,1.0], N=45$; FET, $P<0.0001)$, with no differences in $w$ Pig frequency between

319 males and females in other populations. $w$ Pig frequency in males was relatively low in $2019(p=$ $3200.17[0.02,0.48] ; N=12)$, but fixed in $2020(p=1.0[0.83,1.0] ; N=20)$. We interpret these

321 results as pervasive spatial, and rare temporal and sex-specific, variation in $w$ Pig frequency.

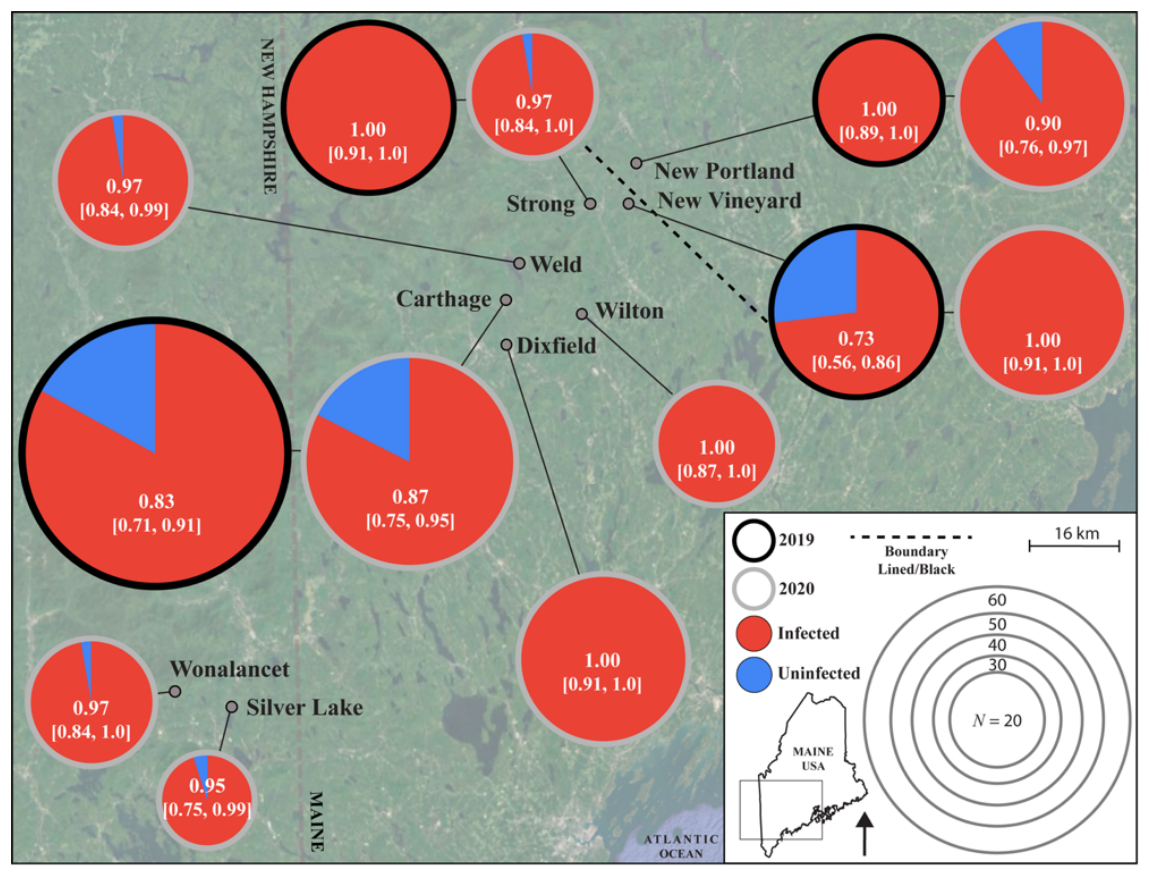

Figure 3. $w$ Pig frequency varies through space and time. Circle size denotes sample size, with outline and fill color denoting sampling year and infection status, respectively. Sample means and $95 \%$ binomial confidence intervals are reported for each sample. The dashed back line denotes the geographical separation of monomorphic black and monomorphic lined $P$. ignipectus populations. 


\section{Discussion}

Our results suggest that $w$ Pig is a group-B Wolbachia acquired after the initial divergence of $P$. ignipectus from P. bicincta. Analysis of Wolbachia and mtDNA haplotypes indicates that

$329 w$ Pig has no apparent effect on the P.ignipectus morphological contact zone in Maine. Across all 330 samples, $w$ Pig occurs at very high frequencies, consistent with our discovery of three divergent 331 sets of CI loci in the $w$ Pig genome. Finally, we document pervasive spatial, and rare temporal, $332 w$ Pig frequency variation. We discuss this in more detail below.

\section{Wolbachia acquisition in spittlebugs}

In contrast to very high $w$ Pig frequencies in P.ignipectus, we found no evidence of Wolbachia in our sample of 100 P. bicincta. A prior report of one infected P. bicincta sample indicates that Wolbachia could infect this species (Anderson, Rustin and Eremeeva, 2019). If so, it must be at very low frequencies, given our credible interval here $(p=0.0[0.0,0.04] ; N=100)$. Mathematical models predict that intense CI drives Wolbachia to high frequencies, balanced by imperfect maternal transmission (Hoffmann, Turelli and Harshman, 1990; Turelli and Hoffmann, 1995); conversely, Wolbachia that do not cause strong CI tend to occur at much lower

342 frequencies (Hamm et al., 2014; Kriesner et al., 2016; Cooper et al., 2017; Hague et al., 2020).

343 While crossing to test for CI in the laboratory is not currently feasible in this system, the

344 presence of three sets of CI loci in the $w$ Pig genome, combined with its very high frequencies, 345 suggests that $w$ Pig causes intense CI.

How did P. ignipectus acquire $w$ Pig? There are three possibilities: cladogenic transmission from its most recent common ancestor with its sister species, presumably $P$.

348 bicincta or a close relative; by introgression from P. bicincta or another close relative; or by

349 horizontal transmission (O’Neill et al., 1992). Given that we find no evidence for a high

350 frequency Wolbachia in P. bicincta, cladogenic acquisition seems implausible. Without more 351 extensive analysis of close relatives, we cannot rule out introgression. However, opportunities 352 for introgression with species other than P. bicincta have likely been limited. Other species of 353 the genus Prosapia or family Cercopidae occur no further north than the USA-Mexico border 354 region, about $1,400 \mathrm{~km}$ from the nearest $P$. ignipectus populations and $3,000 \mathrm{~km}$ from the 355 populations studied here. 
Overall, the limited data are consistent with relatively recent non-cladogenic transmission, a process that seems to be common among Drosophila species (Turelli et al., 2018). It may also be common among spittlebugs. This would be in stark contrast to obligate transovarial endosymbionts associated with amino acid nutrition in spittlebugs and other hemipterans (Koga et al., 2013). In addition to the thrip-related Wolbachia found in P. spumarius in this study, Nakabachi et al. (2020) report that two spittlebug species, Aphrophora quadrinotata Say and Philaenus maghresignus Drosopoulos \& Remane (both Aphrophoridae), harbor Wolbachia with 16S rRNA sequence that is identical to Wolbachia in two psyllid species, two whiteflies, an aphid, a planthopper, two leafhoppers, two grasshoppers, a mosquito and a weevil. Likewise, Lis et al. (2015) report that Wolbachia they studied in P. spumarius is closely related to strains in vespids, drosophilids, whiteflies, chrysomelid beetles and weevils based on five MLST loci. Kapantaidaki et al. (2021) also report Wolbachia infections at low levels in $P$. spumarius, as well as higher frequencies in Neophilaenus campestris (Fallén) (Aphrophoridae). Based on five MLST loci, their N. campestris strain is closely related to Wolbachia found in a leafhopper (Hemiptera) and cluster with Wolbachia from a planthopper, a scale insect and a psyllid (all Hemiptera), as well as two chrysomelid beetles, two butterflies, a parasitic wasp and a mosquito. Koga et al. (2013, table S3) report the presence of Wolbachia in the spittlebug Cosmoscarta heros (F.) (Cercopidae), in addition to A. quadrinotata and P. maghresignus. (Wiwatanaratanabutr, 2015), six specimens of Philaenus tesselatus Melichar (Lis, MaryańskaNadachowska and Kajtoch, 2015), 37 specimens of Philaenus signatus Melichar (Lis, Maryańska-Nadachowska and Kajtoch, 2015; Kapantaidaki et al., 2021), and single specimens of Philaenus arslani Abdul-Nour \& Lahoud, Philaenus loukasi Drosopoulos \& Asche, and Philaenus tarifa Remane \& Drosopoulos (Lis, Maryańska-Nadachowska and Kajtoch, 2015) were not infected. Based on limited sequence data, the emerging pattern suggests that Wolbachia infection is widespread, but far from ubiquitous among spittlebugs, and that when it does occur, it often involves Wolbachia strains similar to those infecting distantly related insects. Whole

383 Wolbachia and host genomic data is sorely needed to test our hypothesis that horizontal

384 Wolbachia acquisition might be common in spittlebugs. 


\section{Little contribution of $w$ Pig to the $P$. ignipectus morphological contact zone}

We find no evidence for differentiation in $w$ Pig or mtDNA haplotypes among $P$. ignipectus color forms. This includes the monomorphic black (New Vineyard) and lined (New Portland) populations that are separated by only $10 \mathrm{~km}$ in Maine, with no obvious barriers to dispersal or reproduction (Thompson and Carvalho, 2016). We also found no variation in $w$ Pig or mtDNA haplotypes between black and lined individuals in the polymorphic Strong population. $w$ Pig frequency also did not vary between color forms. These data indicate that $w$ Pig is unlikely to significantly contribute to the maintenance of the P.ignipectus morphological contact zone.

How common are Wolbachia effects on host RI? Obligate Wolbachia infections in cooccurring D. paulistorum semi-species contribute to assortative mating and generate hybrid inviability and male sterility (Miller, Ehrman and Schneider, 2010). Wolbachia also contribute to reinforcement between Wolbachia-infected D. recens and uninfected D. subquinaria

400 (Shoemaker, Katju and Jaenike, 1999; Jaenike et al., 2006). In contrast, Wolbachia do not 401 contribute to premating, gametic, or postzygotic RI among the three D. yakuba-clade host 402 species (Cooper et al., 2017). While the crossing schemes used in these Drosophila studies to 403 dissect Wolbachia contributions to RI are not feasible in P. ignipectus and many other systems, 404 our genetic data here lend support to our prior conjecture that Wolbachia contributions to RI 405 observed in some Drosophila may be the exception rather than the rule (Turelli, Lipkowitz and 406 Brandvain, 2014; Cooper et al., 2017). effects, and the severity of CI govern Wolbachia frequencies in host populations. Wolbachia that

411 cause intense CI tend to occur at high and stable frequencies, balanced by imperfect maternal 412 transmission (Barton and Turelli, 2011; Turelli and Hoffmann, 1995; Hoffmann, Turelli and 413 Harshman, 1990; Turelli and Hoffmann, 1991; Carrington et al., 2011; Kriesner et al., 2013);

414 while Wolbachia that cause weak or no CI tend to persist at intermediate, often variable 415 frequencies (Hamm et al., 2014; Kriesner et al., 2016; Cooper et al., 2017; Hague et al., 2020). 416 Accumulating evidence for variable infection frequencies (Hamm et al., 2014; Kriesner et al., 417 2016; Schuler et al., 2016; Hughes et al., 2011; Lis, Maryańska-Nadachowska and Kajtoch, 
2015; Cooper et al., 2017), including our discovery here, highlights that infection frequencies are not static, even for high frequency variants. parameters required to approximate population frequency dynamics and equilibria of Wolbachia

422 exist (Turelli and Hoffmann, 1995; Carrington et al., 2011). wMel-like Wolbachia frequencies in 423 the D. yakuba clade vary through space and time in west Africa (Cooper et al., 2017), due in part 424 to effects of cold temperatures on $w$ Yak titer (Hague et al., 2020). CI strength also varies in the 425 D. yakuba clade, which may influence infection frequencies (Cooper et al., 2017; Hague,

426 Caldwell and Cooper, 2020). wMel frequencies vary with latitude in D. melanogaster

427 populations, potentially due to $w \mathrm{Mel}$ fitness costs in the cold (Kriesner et al., 2016).

428 Interestingly, hot temperatures reduce $w \mathrm{Mel} \mathrm{CI}$ strength and transmission in transinfected Aedes 429 aegypti used for biocontrol of human disease (Ross et al., 2017, 2020), suggesting that

430 temperature may generally influence key parameters underlying Wolbachia infection 431 frequencies.

432 What underlies variable $w$ Pig frequencies in nature? High $w$ Pig frequencies and the 433 presence of three divergent sets of cifs suggest, but do not confirm, that $w$ Pig causes strong CI. It 434 seems plausible that some or all of these loci were horizontally acquired (Cooper et al., 2019), 435 but additional sequence data are required to test this. We hypothesize that variable $w$ Pig 436 transmission rates contribute to the frequency variation we observe, potentially due to 437 environmental effects on titer, as observed for $w$ Yak (Hague et al., 2020). Temporal variation in 438 transmission was also observed for $w \mathrm{Ri}$ between two samples of $D$. simulans collected from 439 Ivanhoe, California in April and November of 1993 (Turelli and Hoffmann, 1995; Carrington et 440 al., 2011), although the relative stability of $w$ Ri frequencies in global $D$. simulans populations

441 suggests that its transmission persists across a range of environmental conditions. Additional 442 analyses of Wolbachia titer and transmission in the field, and across environmental contexts, are 443 needed to better understand the causes of Wolbachia frequency variation. Comparing the titer 444 and transmission of Wolbachia that occur at different frequencies in nature-for example, those 445 that do and do not cause intense CI-would be particularly useful.

\section{Data Accessibility Statement}

448 All data will be uploaded to DRYAD or GenBank upon acceptance. 


\section{Competing Interests Statement}

450 We declare no competing interests.

\section{Author Contributions Section}

453 Timothy B. Wheeler: Data curation, Investigation, Validation, Visualization, Writing - original

454 draft, Writing - review \& editing. Vinton Thompson: Conceptualization, Data curation, Formal

455 analysis, Investigation, Methodology, Project administration, Resources, Visualization, Writing -

456 original draft, Writing - review \& editing. William R. Conner: Data curation, Formal analysis,

457 Investigation, Writing - original draft, Writing - review \& editing. Brandon S. Cooper:

458 Conceptualization, Data curation, Formal analysis, Funding acquisition, Investigation,

459 Methodology, Project administration, Resources, Supervision, Validation, Visualization, Writing

460 - original draft, Writing - review \& editing.

\section{Acknowledgments}

463 We thank M. Thorne and A.G. Dale for P. bicincta collections and D. McVicar and F. Selchin

464 for access to the Wonaloncet site. Michael Turelli provided comments that greatly improved an

465 earlier draft. We also thank M. Hague, D. Shropshire, and K. Van Vaerenberghe for very helpful

466 comments. Research reported in this publication was supported by the National Institute of

467 General Medical Sciences of the National Institutes of Health (NIH) under award number

468 R35GM124701 to B.S.C., and by the University of Montana Genomics Core. 


\section{References}

Ahmed, M. Z. et al. (2015) 'The intracellular bacterium Wolbachia uses parasitoid wasps as phoretic vectors for efficient horizontal transmission', PLoS Pathogens, 11(2), p. e1004672. doi: 10.1371/journal.ppat.1004672.

Altschul, S. F. et al. (1990) 'Basic local alignment search tool', Journal of Molecular Biology, 215(3), pp. 403-410. doi: 10.1016/S0022-2836(05)80360-2.

Anderson, M. L., Rustin, R. C. and Eremeeva, M. E. (2019) 'Pilot survey of mosquitoes (Diptera: Culicidae) from southeastern Georgia, USA for Wolbachia and Rickettsia felis (Rickettsiales: Rickettsiaceae)', Journal of Vector Borne Diseases, 56(2), pp. 92-97. doi: 10.4103/0972-9062.263714.

491 Baldo, L. et al. (2006) 'Multilocus sequence typing system for the endosymbiont Wolbachia pipientis', Applied and environmental microbiology, 72(11), pp. 7098-7110.

Bandi, C. et al. (1998) 'Phylogeny of Wolbachia in filarial nematodes', Proceedings of the Royal Society of London. Series B: Biological Sciences, 265(1413), pp. 2407-2413.

495 Barton, N. and Turelli, M. (2011) 'Spatial waves of advance with bistable dynamics: cytoplasmic and genetic analogues of Allee effects', The American Naturalist, 178(3), pp. E48-E75.

Beckmann, J. F., Ronau, J. A. and Hochstrasser, M. (2017) 'A Wolbachia deubiquitylating enzyme induces cytoplasmic incompatibility’, Nature Microbiology, 2(5), p. 17007.

Bing, X.-L. et al. (2020) 'Genomic analysis of Wolbachia from Laodelphax striatellus (Delphacidae, Hemiptera) reveals insights into its "Jekyll and Hyde" mode of infection pattern', Genome Biology and Evolution, 12(2), pp. 3818-3831. doi: 10.1093/gbe/evaa006.

Braig, H. R. et al. (1998) 'Cloning and characterization of a gene encoding the major surface protein of the bacterial endosymbiont Wolbachia pipientis', Journal of Bacteriology, 180(9), pp.

Brownlie, J. C. et al. (2009) 'Evidence for metabolic provisioning by a common invertebrate endosymbiont, Wolbachia pipientis, during periods of nutritional stress', PLoS Pathogens, 5(4), p. e1000368.

508 Carrington, L. B. et al. (2011) 'A re-examination of Wolbachia-induced cytoplasmic

509 incompatibility in California Drosophila simulans', PLoS One, 6(7), p. e22565.

510 Carvalho, G. S. and Webb, M. D. (2005) Cercopid spittle bugs of the New World: Hemiptera,

511 Auchenorrhyncha, Cercopidae. 1. ed. Sofía Moscow: Pensoft (Pensoft series faunistica, 49).

512 Caspari, E. and Watson, G. (1959) 'On the evolutionary importance of cytoplasmic sterility in 513 mosquitoes', Evolution, 13(4), pp. 568-570. 
514 Cattel, J. et al. (2016) 'Wolbachia in European populations of the invasive pest Drosophila

515 suzukii: regional variation in infection frequencies', PLoS One, 11(1), p. e0147766.

516 Chrostek, E. et al. (2017) 'Horizontal transmission of intracellular insect symbionts via plants',

517 Frontiers in Microbiology, 8. doi: 10.3389/fmicb.2017.02237.

518 Conner, W. R. et al. (2017) 'Genome comparisons indicate recent transfer of $w$ Ri-like

519 Wolbachia between sister species Drosophila suzukii and D. subpulchrella', Ecology and

520 Evolution, 7(22), pp. 9391-9404.

521 Cooper, B. S. et al. (2017) 'Wolbachia in the Drosophila yakuba complex: pervasive frequency

522 variation and weak cytoplasmic incompatibility, but no apparent effect on reproductive

523 isolation', Genetics, 205(1), pp. 333-351.

524 Cooper, B. S. et al. (2019) 'Wolbachia acquisition by Drosophila yakuba-clade hosts and

525 transfer of incompatibility loci between distantly related Wolbachia', Genetics, 212(4), pp.

526 1399-1419.

527 Coyne, J. A. and Orr, H. A. (2004) Speciation. Sunderland, Massachusetts: Sinauer Associates.

Fagan, E. B. and Kuitert, L. C. (1969) 'Biology of the Two-Lined Spittlebug, Prosapia bicincta, on Florida Pastures (Homoptera: Cercopidae)', The Florida Entomologist, 52(3), pp. 199-206.

531 Foottit, R. G., Maw, E. and Hebert, P. D. N. (2014) 'DNA barcodes for Nearctic

532 Auchenorrhyncha (Insecta: Hemiptera)', PloS One, 9(7), p. e101385. doi:

533 10.1371/journal.pone.0101385.

Fredericksen, M. A. et al. (2017) 'Three-dimensional visualization and a deep-learning model reveal complex fungal parasite networks in behaviorally manipulated ants', Proceedings of the National Academy of Sciences, 114(47), pp. 12590-12595. doi: 10.1073/pnas.1711673114.

Gerth, M. and Bleidorn, C. (2017) 'Comparative genomics provides a timeframe for Wolbachia evolution and exposes a recent biotin synthesis operon transfer', Nature Microbiology, 2(3), p. 16241.

Gould, A. L. et al. (2018) 'Microbiome interactions shape host fitness', Proceedings of the

542 Hague, M. T. et al. (2020) 'Environmental and genetic contributions to imperfect $w$ Mel-like 543 Wolbachia transmission and frequency variation', Genetics, 215(4), pp. 1117-1132.

544 Hague, M. T., Caldwell, C. N. and Cooper, B. S. (2020) 'Pervasive effects of Wolbachia on host 545 temperature preference', mBio, 11(5). 
Hamilton, K. G. A. (1982) The spittlebugs of Canada: Homoptera--Cercopidae. Ottawa, Ont: Biosystematics Research Institute : Canadian Govt. Pub. Centre, Supply and Services Canada [distributor] (The Insects and arachnids of Canada, pt. 10).

Hamm, C. A. et al. (2014) 'Wolbachia do not live by reproductive manipulation alone: infection polymorphism in Drosophila suzukii and D. subpulchrella', Molecular Ecology, 23(19), pp. 4871-4885.

Hoffmann, A. A., Clancy, D. and Duncan, J. (1996) 'Naturally-occurring Wolbachia infection in Drosophila simulans that does not cause cytoplasmic incompatibility', Heredity, 76(1), pp. 1-8.

Hoffmann, A. A. and Turelli, M. (1997) 'Cytoplasmic incompatibility in insects', in O’Neill, S. L., Hoffmann, A. A., and Werren, J. H. (eds) Influential passengers: inherited microorganisms and arthropod reproduction. Oxford University Press, pp. 42-80.

560 Hoffmann, A. A., Turelli, M. and Harshman, L. G. (1990) 'Factors affecting the distribution of cytoplasmic incompatibility in Drosophila simulans.', Genetics, 126(4), pp. 933-948.

Höhna, S. et al. (2016) 'RevBayes: Bayesian phylogenetic inference using graphical models and an interactive model-specification language', Systematic Biology, 65(4), pp. 726-736.

Hughes et al. (2011) 'Variable infection frequency and high diversity of multiple strains of Wolbachia pipientis in Perkinsiella planthoppers', Applied and Environmental Microbiology, 77(6), pp. 2165-2168. doi: 10.1128/AEM.02878-10.

567 Hughes, G. et al. (2011) 'Wolbachia infections in Anopheles gambiae cells: transcriptomic 568 characterization of a novel host-symbiont interaction', PLoS pathogens, 7(2), p. e1001296.

Huigens, M. E. et al. (2000) 'Infectious parthenogenesis', Nature, 405(6783), pp. 178-179. doi: 10.1038/35012066.

Hurst, G. D. and Jiggins, F. M. (2000) 'Male-killing bacteria in insects: mechanisms, incidence, 572 and implications.', Emerging Infectious Diseases, 6(4), pp. 329-336.

573 Jaenike, J. et al. (2006) 'Asymmetrical Reinforcement and Wolbachia Infection in Drosophila', 574 PLoS Biology, 4(10), p. e325. doi: 10.1371/journal.pbio.0040325.

575 Kapantaidaki, D. E. et al. (2021) 'Genetic and endosymbiotic diversity of Greek populations of 576 Philaenus spumarius, Philaenus signatus and Neophilaenus campestris, vectors of Xylella 577 fastidiosa', Scientific Reports, 11(1), p. 3752. doi: 10.1038/s41598-021-83109-z.

578 Klasson, L. et al. (2008) 'Genome evolution of Wolbachia strain wPip from the Culex pipiens 579 group', Molecular Biology and Evolution, 25(9), pp. 1877-1887. doi: 10.1093/molbev/msn133.

580 Klasson, L. et al. (2009) 'The mosaic genome structure of the Wolbachia wRi strain infecting 581 Drosophila simulans', Proceedings of the National Academy of Sciences, 106(14), pp. 57255825730. 
Koga, R. et al. (2013) 'Evolutionary replacement of obligate symbionts in an ancient and diverse insect lineage', Environmental Microbiology, 15(7), pp. 2073-2081. doi:

585 https://doi.org/10.1111/1462-2920.12121.

586

587

588

Koren, S. et al. (2017) 'Canu: scalable and accurate long-read assembly via adaptive k-mer weighting and repeat separation', Genome Research, p. gr.215087.116. doi: 10.1101/gr.215087.116.

Koren, S. et al. (2018) 'De novo assembly of haplotype-resolved genomes with trio binning', Nature Biotechnology, 36(12), pp. 1174-1182. doi: 10.1038/nbt.4277.

Kriesner, P. et al. (2013) 'Rapid sequential spread of two Wolbachia variants in Drosophila simulans', PLoS Pathogens, 9(9), p. e1003607.

Kriesner, P. et al. (2016) 'Persistence of a Wolbachia infection frequency cline in Drosophila melanogaster and the possible role of reproductive dormancy', Evolution, 70(5), pp. 979-997.

LePage, D. P. et al. (2017) 'Prophage WO genes recapitulate and enhance Wolbachia-induced cytoplasmic incompatibility', Nature, 543(7644), p. 243.

Lindsey, A. R. I. et al. (2018) 'Evolutionary genetics of cytoplasmic incompatibility genes cifA and cifB in prophage WO of Wolbachia', Genome Biology and Evolution, 10(2), pp. 434-451. doi: 10.1093/gbe/evy012.

Lis, A., Maryańska-Nadachowska, A. and Kajtoch, Ł. (2015) 'Relations of Wolbachia infection with phylogeography of Philaenus spumarius (Hemiptera: Aphrophoridae) populations within and beyond the Carpathian contact zone', Microbial Ecology, 70(2), pp. 509-521. doi: 10.1007/s00248-015-0570-2.

Loman, N. J., Quick, J. and Simpson, J. T. (2015) 'A complete bacterial genome assembled de novo using only nanopore sequencing data', Nature Methods, 12(8), pp. 733-735. doi: 10.1038/nmeth.3444.

Martinez, J. et al. (2020) 'Life and death of selfish genes: comparative genomics reveals the dynamic evolution of cytoplasmic incompatibility', Molecular Biology and Evolution, (msaa209). doi: 10.1093/molbev/msaa209.

Matute, D. R. and Cooper, B. S. (2021) 'Comparative studies on speciation: 30 years since Coyne and Orr', Evolution; International Journal of Organic Evolution. doi: 10.1111/evo.14181.

McCutcheon, J. P., Boyd, B. M. and Dale, C. (2019) 'The life of an insect endosymbiont from the cradle to the grave', Current Biology, 29(11), pp. R485-R495. doi: 10.1016/j.cub.2019.03.032.

McFall-Ngai, M. et al. (2013) 'Animals in a bacterial world, a new imperative for the life sciences', Proceedings of the National Academy of Sciences, 110(9), pp. 3229-3236. doi: 10.1073/pnas.1218525110. 
Meany, M. K. et al. (2019) 'Loss of cytoplasmic incompatibility and minimal fecundity effects explain relatively low Wolbachia frequencies in Drosophila mauritiana', Evolution, 73(6), pp.

$620 \quad 1278-1295$.

621 Miller, W. J., Ehrman, L. and Schneider, D. (2010) 'Infectious speciation revisited: impact of 622 symbiont-depletion on female fitness and mating behavior of Drosophila paulistorum', PLoS

623 Pathogens. Edited by C. Parrish, 6(12), p. e1001214. doi: 10.1371/journal.ppat.1001214.

624 Nakabachi, A. et al. (2020) '16S rRNA Sequencing Detected Profftella, Liberibacter,

625 Wolbachia, and Diplorickettsia from Relatives of the Asian Citrus Psyllid', Microbial Ecology,

626 80(2), pp. 410-422. doi: 10.1007/s00248-020-01491-z.

627 Nurk, S. et al. (2020) 'HiCanu: accurate assembly of segmental duplications, satellites, and 628 allelic variants from high-fidelity long reads', Genome Research, 30(9), pp. 1291-1305. doi:

$62910.1101 /$ gr.263566.120.

630 O'Neill, S. L. et al. (1992) '16S rRNA phylogenetic analysis of the bacterial endosymbionts 631 associated with cytoplasmic incompatibility in insects', Proceedings of the National Academy of 632 Sciences, 89(7), pp. 2699-2702.

633 Peck, D. C. (1999) 'Seasonal fluctuations and phenology of Prosapia Spittlebugs (Homoptera: 634 Cercopidae) in upland pastures of Costa Rica', Environmental Entomology, 28(3), pp. 372-386. 635 doi: 10.1093/ee/28.3.372.

636 Rambaut, A. et al. (2014) Tracer. Available at: http://beast.bio.ed.ac.uk/Tracer.

637 Raychoudhury, R. et al. (2009) 'Modes of acquisition of Wolbachia: horizontal transfer, hybrid 638 introgression, and codivergence in the Nasonia species complex’, Evolution, 63(1), pp. $165-183$.

639 Ross, P. A. et al. (2017) 'Wolbachia infections in Aedes aegypti differ markedly in their response to cyclical heat stress', PLoS Pathogens, 13(1), p. e1006006.

641 Ross, P. A. et al. (2020) 'Heatwaves cause fluctuations in wMel Wolbachia densities and 642 frequencies in Aedes aegypti', PLoS Neglected Tropical Diseases, 14(1), p. e0007958. doi:

643 10.1371/journal.pntd.0007958.

644 Schuler, H. et al. (2016) 'The hitchhiker's guide to Europe: the infection dynamics of an ongoing 645 Wolbachia invasion and mitochondrial selective sweep in Rhagoletis cerasi', Molecular 646 Ecology, 25(7), pp. 1595-1609.

647 Seemann, T. (2014) 'Prokka: rapid prokaryotic genome annotation', Bioinformatics, 30(14), pp. $648 \quad 2068-2069$.

649 Shoemaker, D. D., Katju, V. and Jaenike, J. (1999) 'Wolbachia and the evolution of reproductive 650 isolation between Drosophila recens and Drosophila subquinaria', Evolution; International 651 Journal of Organic Evolution, 53(4), pp. 1157-1164. doi: 10.1111/j.1558-5646.1999.tb04529.x. 
652 Shropshire, J. D. et al. (2018) 'One prophage WO gene rescues cytoplasmic incompatibility in

653 Drosophila melanogaster’, Proceedings of the National Academy of Sciences, 115(19), pp.

$654 \quad 4987-4991$.

655 Shropshire, J. D. and Bordenstein, S. R. (2019) 'Two-By-One model of cytoplasmic 656 incompatibility: Synthetic recapitulation by transgenic expression of cifA and cifB in 657 Drosophila', PLoS Genetics, 15(6), p. e1008221. doi: 10.1371/journal.pgen.1008221.

658 Shropshire, J. D., Leigh, B. and Bordenstein, S. R. (2020) 'Symbiont-mediated cytoplasmic 659 incompatibility: what have we learned in 50 years?', eLife. Edited by D. Weigel, 9, p. e61989. 660 doi: 10.7554/eLife.61989.

661 Thompson, V. (2004) 'Associative nitrogen fixation, $\mathrm{C}_{4}$ photosynthesis, and the evolution of 662 spittlebugs (Hemiptera: Cercopidae) as major pests of neotropical sugarcane and forage grasses', 663 Bulletin of Entomological Research, 94(3), pp. 189-200. doi: 10.1079/BER2004293.

664 Thompson, V. and Carvalho, G. S. (2016) 'Abrupt geographical transition between aposematic 665 color forms in the spittlebug Prosapia ignipectus (Fitch)(Hemiptera: Cercopidae).', Psyche, 666 2016, p. e3623092. doi: https://doi.org/10.1155/2016/3623092.

667 Thorne, M. S. et al. (2018) 'Two-lined Spittlebug (Prosapia bicincta (Say)) in Hawaii’, p. 3.

668 Toju, H. et al. (2013) 'Diversification of endosymbiosis: replacements, co-speciation and 669 promiscuity of bacteriocyte symbionts in weevils', The ISME Journal, 7(7), pp. 1378-1390. doi: $670 \quad 10.1038 /$ ismej.2013.27.

671 Turelli, M. et al. (2018) 'Rapid global spread of $w$ Ri-like Wolbachia across multiple 672 Drosophila', Current Biology, 28(6), pp. 963-971.e8.

673 Turelli, M. and Hoffmann, A. A. (1991) 'Rapid spread of an inherited incompatibility factor in 674 California Drosophila', Nature, 353(6343), pp. 440-442.

675 Turelli, M. and Hoffmann, A. A. (1995) 'Cytoplasmic incompatibility in Drosophila simulans: 676 Dynamics and parameter estimates from natural populations’, Genetics, 140(4), pp. 1319-1338.

677 Turelli, M., Lipkowitz, J. R. and Brandvain, Y. (2014) 'On the Coyne and Orr-Igin of Species: 678 Effects of intrinsic postzygotic isolation, ecological differentiation, $\mathrm{X}$ chromosome size, and 679 sympatry on Drosophila speciation', Evolution, 68(4), pp. 1176-1187. doi:

680 https://doi.org/10.1111/evo.12330.

681 Weinert, L. A. et al. (2015) 'The incidence of bacterial endosymbionts in terrestrial arthropods', 682 Proceedings of the Royal Society B: Biological Sciences, 282(1807), p. 20150249.

683 Werren, J. H., Baldo, L. and Clark, M. E. (2008) 'Wolbachia: master manipulators of 684 invertebrate biology’, Nature Reviews Microbiology, 6(10), pp. 741-751. 
685 Wiwatanaratanabutr, I. (2015) 'Wolbachia infection in leafhoppers and planthoppers: Diversity, 686 density and geographic distribution in tropical rice agroecosystems', Journal of Asia-Pacific

687 Entomology, 18(2), pp. 277-282. doi: 10.1016/j.aspen.2015.03.004.

688 Wu, M. et al. (2004) 'Phylogenomics of the reproductive parasite Wolbachia pipientis wMel: a 689 streamlined genome overrun by mobile genetic elements', PLoS biology, 2(3), p. e69.

690 Yang, Z. (1994) 'Maximum likelihood phylogenetic estimation from DNA sequences with 691 variable rates over sites: Approximate methods', Journal of Molecular Evolution, 39(3), pp. 692 306-314. doi: 10.1007/BF00160154.

693 Zug, R. and Hammerstein, P. (2012) 'Still a host of hosts for Wolbachia: analysis of recent data 694 suggests that $40 \%$ of terrestrial arthropod species are infected', PLoS One, 7(6), p. e38544. 
bioRxiv preprint doi: https://doi.org/10.1101/2021.02.25.432892; this version posted February 25, 2021. The copyright holder for this preprint (which was not certified by peer review) is the author/funder, who has granted bioRxiv a license to display the preprint in perpetuity. It is made available under aCC-BY-ND 4.0 International license.

Supplemental Table 1. Spittlebugs from Schizachyrium scoparium, Maine and New Hampshire, August 2019 and 2020

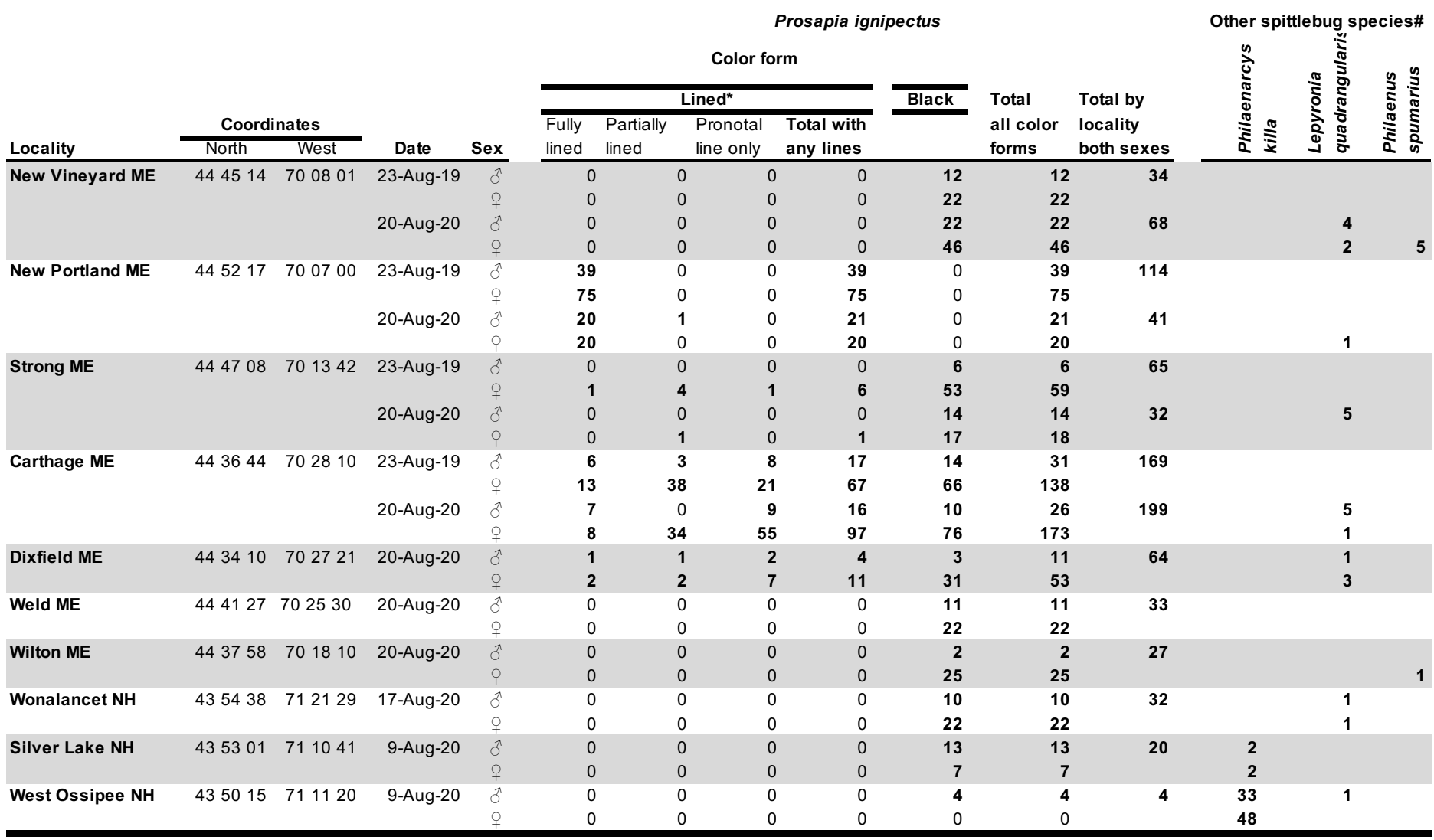

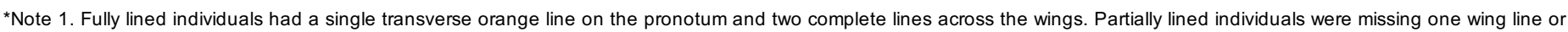
had one or two interrupted or incomplete wing lines. Pronotum line only individuals lacked wing lines but possessed the pronotal line in full or in partially obscured form.

\#Note 2. Other spittlebug species are recorded only for the 2020 collections, which included all specimens in this category tested for the presence of Wolbachia. 
Supplemental Table 2. PCR primers used in this study

\begin{tabular}{|c|c|c|}
\hline Primer Name & Sequence (5'-- 3') & Reference \\
\hline \multicolumn{3}{|c|}{ Mitochondrial CoI } \\
\hline LepF & 5'-ATTCAACCAATCATAAAGATATTGG-3' & Hebert et al. (2004a) \\
\hline LepR & 5'-TAAACTTCTGGATGTCCAAAAAATCA-3' & Hebert et al. (2004a) \\
\hline \multicolumn{3}{|c|}{ MLST } \\
\hline $\operatorname{cox} A \_F 1$ & 5'-TTGGRGCRATYAACTTTATAG-3' & Baldo et al. 2006 \\
\hline $\operatorname{cox} A \_R 1$ & 5'-CTAAAGACTTTKACRCCAGT-3' & Baldo et al. 2006 \\
\hline$g a t B \_F 1$ & 5'-GAKTTAAAYCGYGCAGGBGTT-3' & Baldo et al. 2006 \\
\hline gatB_Rl & 5'-TGGYAAYTCRGGYAAAGATGA-3' & Baldo et al. 2006 \\
\hline$h c p A_{-} F 1$ & 5'-GAAATARCAGTTGCTGCAAA-3' & Baldo et al. 2006 \\
\hline$h c p A \_R l$ & 5'-GAAAGTYRAGCAAGYTCTG-3' & Baldo et al. 2006 \\
\hline$f b p A \_F 1$ & 5'-GCTGCTCCRCTTGGYWTGAT-3' & Baldo et al. 2006 \\
\hline$f b p A-R 1$ & 5'-CCRCCAGARAAAAYYACTATTC-3' & Baldo et al. 2006 \\
\hline$f b p A \_F 3$ & 5'-GTTAACCCTGATGCYYAYGAYCC-3' & Baldo et al. 2006 \\
\hline$f b p A \_R 3$ & 5'-TCTACTTCCTTYGAYTCDCCRCC-3' & Baldo et al. 2006 \\
\hline$w s p \_F 1$ & 5'-GTCCAATARSTGATGARGAAAC-3' & Baldo et al. 2006 \\
\hline$w s p \_R l$ & 5'-CYGCACCAAYAGYRCTRTAAA-3' & Baldo et al. 2006 \\
\hline ftsZunif & 5'-GGYAARGGTGCRGCAGAAGA-3' & Lo et al. 2002 \\
\hline ftsZunir & 5'-ATCRATRCCAGTTGCAAG-3' & Lo et al. 2002 \\
\hline \multicolumn{3}{|c|}{ Wolbachia infection screen } \\
\hline$w s p \_p c r \_F$ & 5'-TGGTCCAATAAGTGATGAAGAAAC-3' & Braig et al. 1998 \\
\hline wsp_pcr_R & 5'-AAAAATTAAACGCTACTCCA-3' & Braig et al. 1998 \\
\hline $28 s \_p c r \_F$ & 5'-TACCGTGAGGGAAAGTTGAAA-3' & Werren et al. 1995 \\
\hline $28 s \_p c r \_R$ & 5'-AGACTCCTTGGTCCGTGTTT-3' & Werren et al. 1995 \\
\hline
\end{tabular}

